\title{
Optimal Analysis of a Communication Network Model
}

\author{
N. Thirupathi Rao ${ }^{1}$ and Debnath Bhattacharyya ${ }^{2}$ \\ Department of Computer Science and Engineering, Vignan's Institute of Information \\ Technology (A), Visakhapatnam-530049, AP, India, \\ 'nakkathiru@gmail.co, ${ }^{2}$ debnathb@gmail.com
}

\begin{abstract}
This paper tends to the original thought of using compound Poisson binomial process for creating and investigating a two-hub couple communication coordinate with two-phase landings and Dynamic Bandwidth Allocation (DBA). Here it is accepted that two hubs are associated pair and messages touch base to the first and second supports are associated with an irregular number of bundles and put away in cradles for forward transmission. Entries are portrayed by compound Poisson binomial procedures in the two cradles which coordinate close with the reasonable circumstance. The transmission forms in both the transmitters are expected to take after unique transfer speed allotment which is portrayed by stack reliant on time. Utilizing distinction differential conditions and joint likelihood producing capacity the transient conduct of the framework is examined. The execution of the system is assessed by determining unequivocal articulations for the execution measures, for example, mean substance of the cradles, mean postponements, throughput of the hubs and usage of transmitters. Numerical representations are displayed to examine the impact of changes in input parameters on framework execution measures. With reasonable cost contemplations, the ideal working strategies of the communication networks are determined and broke down. It is watched that the compound Poisson binomial mass landings dissemination parameters have noteworthy impact on framework execution measures. Dissecting the two-phase coordinate landings enhances the system execution and diminish clog in cradles and mean postponements.
\end{abstract}

Keywords: Communication networks, DBA, Queuing models, Bandwidth, Throughput, Optimal values, Optimization, Sensitivity

\section{Introduction}

Communication networks displaying is an essential for the plan and investigation of numerous communication frameworks. It is hard to lead research centre examinations under factor stack conditions, the communication organize models are created with different suspicions on landing forms, transmission forms, designation, steering and stream control systems [1][2]. For better usage of assets and to enhance nature of administration parcel exchanging is utilized over-circuit or message exchange. Much work has been accounted for in writing in regards to communication systems with blockage control procedures. Bit dropping is one of the typical strategies received for blockage control. In this technique, the thought is disposing of certain segment of benefit, for example, minimum huge bits with a specific end goal to diminish the heap. In any case, the bit dropping makes changes in voice quality because of a progressively

Article history:

Received (January 16, 2019), Review Result (March 6, 2019), Accepted (May 19, 2019) 
fluctuating piece-rate amid a cell transmission. To keep up nature of administration and to lessen the clog in cradles another transmission system dynamic data transfer capacity designation technique is used as an option and effective control procedure.

In every one of the papers alluded above, they expected that the landings are single and take after Poisson process. In any case, in packetized exchanging the message that touches base to the source are changed over into an arbitrary number of parcels and land to the cushions in mass. In any case, in these papers likewise the creators considered that the entries to the system are to be first cradle as it were [3]. Yet, in some communication frameworks like satellite and remote interchanges, there is a two-phase landing i.e., the entries of bundles are to the principal cradle and furthermore to the second support specifically. For instance, in media communications there are some neighborhood calls and some STD calls where the STD calls may straightforwardly touch base to the second cushion. To break down this kind of frameworks, a two hub pair communication networks coordinate with dynamic data transfer capacity allotment having two-phase coordinate compound binomial Poisson entries is produced and broke down.

\subsection{Queuing model}

Consider two-transmitters pair correspondence organizes in which the messages touch base to the system are changed over into an irregular number of bundles [4]. The landing procedure of the messages is arbitrary and various parcels $(\mathrm{X})$ that a message can be changed overtakes after a binomial dissemination with parameters $\mathrm{m}$ and $\mathrm{p}$.

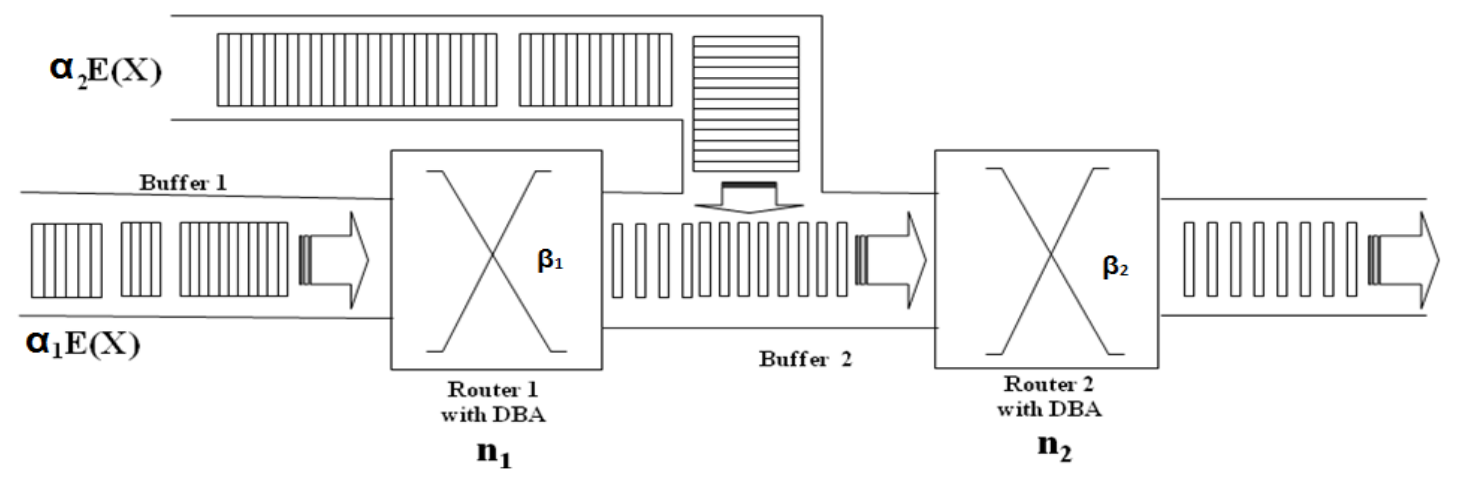

Figure 1. Communication network with two stage bulk arrivals

\section{Mathematical design of the considered model}

In this segment, we infer the ideal working arrangements of the correspondence organizes under investigation [5][6]. Here, it is expected that the specialist co-op of the correspondence arrange is keen on augmentation of the benefit work at a given time t. Let the specialist co-op gets a measure of Ri units per each unit of time of the framework occupied at ith transmitter $(i=1,2)$. As it were, he gets income of Ri units per each unit of throughput of the ith transmitter. In this manner, the aggregate income of the correspondence arrange at time $t$ is,

$R(t)=R_{1}$. (Number of packets transmitting through transmitter 1$)+R_{2}$. (Number of packets transmitting through transmitter 2) 


$$
\begin{aligned}
& \mathrm{R}(\mathrm{t})=\mathrm{R}_{1} \cdot \beta_{1}\left[1-\exp \left[\alpha_{1} \sum_{\mathrm{k}_{1}=1}^{\mathrm{m}_{1}} \sum_{\mathrm{r}=1}^{\mathrm{k}_{1}} \frac{{ }^{\mathrm{m}_{1}} \mathrm{C}_{\mathrm{k}_{1}} \mathrm{p}_{1}{ }^{\mathrm{k}_{1}}\left(1-\mathrm{p}_{1}\right)^{\mathrm{m}_{1}-\mathrm{k}_{1}}}{1-\left(1-\mathrm{p}_{1}\right)^{\mathrm{m}_{1}}}{ }^{\mathrm{k}_{1}} \mathrm{C}_{\mathrm{r}}(-1)^{3 \mathrm{r}} \frac{\left(1-\mathrm{e}^{-\mathrm{r} \beta_{1} \mathrm{t}}\right)}{\mathrm{r} \beta_{1}}\right]\right] \\
& +\mathrm{R}_{2} \cdot \beta_{2} \cdot\left\{1-\exp \left\{\left[\alpha_{1} \sum_{\mathrm{k}_{1}=1}^{\mathrm{m}_{1}} \sum_{\mathrm{r}=1}^{\mathrm{k}_{1}} \sum_{\mathrm{J}=0}^{\mathrm{r}}(-1)^{3 \mathrm{r}-\mathrm{J}} \frac{{ }^{\mathrm{m}_{1}} \mathrm{C}_{\mathrm{k}_{1}} \mathrm{p}_{1}^{\mathrm{k}_{1}}\left(1-\mathrm{p}_{1}\right)^{\mathrm{m}_{1}-\mathrm{k}_{1}}}{1-\left(1-\mathrm{p}_{1}\right)^{\mathrm{m}_{1}}}\left({ }^{\mathrm{k}_{1}} \mathrm{C}_{\mathrm{r}}\right)\left({ }^{\mathrm{r}} \mathrm{C}_{\mathrm{J}}\right)\left(\frac{\beta_{1}}{\beta_{2}-\beta_{1}}\right)^{\mathrm{r}}\left(\frac{1-\mathrm{e}^{-\left[\mathrm{I} \beta_{2}+(\mathrm{r}-\mathrm{J}) \beta_{1}\right] \mathrm{t}}}{\mathrm{J} \beta_{2}+(\mathrm{r}-\mathrm{J}) \beta_{1}}\right)\right]\right.\right. \\
& \left.\left.+\alpha_{2}\left[\sum_{\mathrm{k}_{2}=1}^{\mathrm{m}_{2}} \sum_{\mathrm{s}=1}^{\mathrm{k}_{2}} \frac{\mathrm{m}_{2} \mathrm{C}_{\mathrm{k}_{2}} \mathrm{p}_{2}{ }^{\mathrm{k}_{2}}\left(1-\mathrm{p}_{2}\right)^{\mathrm{m}_{2}-\mathrm{k}_{2}}}{1-\left(1-\mathrm{p}_{2}\right)^{\mathrm{m}_{2}}}\left({ }^{\mathrm{k}_{2}} \mathrm{C}_{\mathrm{s}}\right)(-1)^{\mathrm{S}}\left(\frac{1-\mathrm{e}^{-\mu_{2} \mathrm{k}_{2} \mathrm{t}}}{\mu_{2} \mathrm{k}_{2}}\right)\right]\right\}\right\}
\end{aligned}
$$

$C(t)=A-C_{1} *($ standard waiting time of a client in transmitter 1$)-C_{2} *($ standard waiting time of a client in transmitter 2$)$

$$
\begin{aligned}
& \frac{\frac{\alpha_{1}}{\beta_{1}}\left[\sum_{\mathrm{k}_{1}=1}^{\mathrm{m}_{1}} \frac{{ }^{\mathrm{m}_{1}} \mathrm{C}_{\mathrm{k}_{1}} \mathrm{p}_{1}{ }^{\mathrm{k}_{1}}\left(1-\left(1-\mathrm{p}_{1}\right)^{\mathrm{m}_{1}-\mathrm{k}_{1}}\right.}{1-\left(1-\mathrm{p}_{1}\right)^{\mathrm{m}_{1}}} \cdot \mathrm{k}_{1}\left(1-\mathrm{e}^{-\beta_{1} \mathrm{t}}\right)\right]}{\left.\left[\alpha_{1} \sum_{\mathrm{k}_{1}=1}^{\mathrm{m}_{1}} \sum_{\mathrm{r}=1}^{\mathrm{k}_{1}} \frac{{ }^{\mathrm{m}_{1}} \mathrm{C}_{\mathrm{k}_{1}} \mathrm{p}_{1}{ }^{\mathrm{k}_{1}}\left(1-\mathrm{p}_{1}\right)^{\mathrm{m}_{1}-\mathrm{k}_{1}}}{1-\left(1-\mathrm{p}_{1}\right)^{\mathrm{m}_{1}}}{ }^{\mathrm{k}_{1}} \mathrm{C}_{\mathrm{r}}(-1)^{3 \mathrm{r}} \frac{\left(1-\mathrm{e}^{-\mathrm{r} \beta_{1} \mathrm{t}}\right)}{\mathrm{r} \beta_{1}}\right]\right]}
\end{aligned}
$$

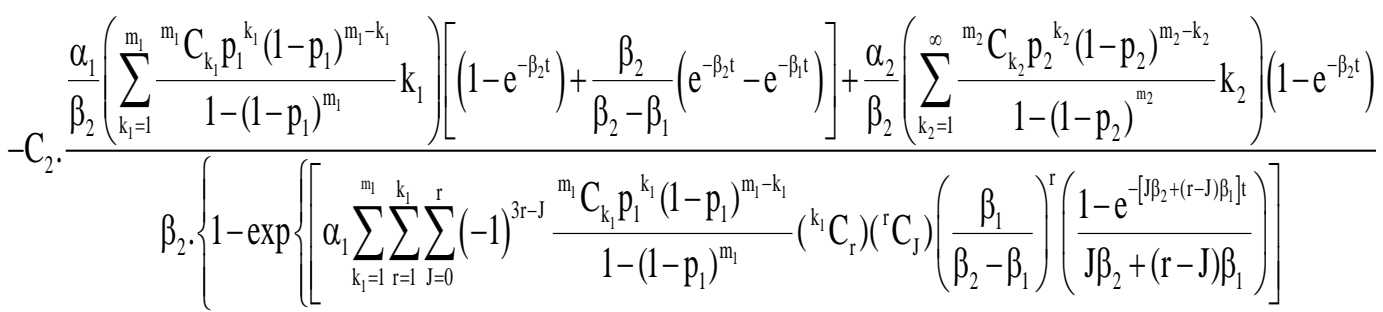

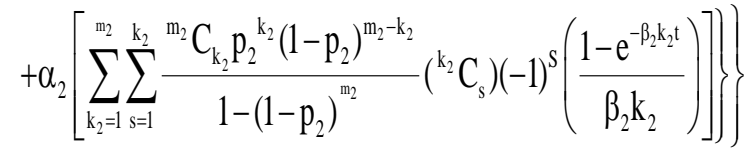

$$
\begin{aligned}
& \mathrm{P}(\mathrm{t})=\mathrm{R}_{1} \cdot \beta_{1}\left[1-\exp \left[\alpha_{1} \sum_{\mathrm{k}_{1}=1}^{\mathrm{m}_{1}} \sum_{\mathrm{r}=1}^{\mathrm{k}_{1}} \frac{{ }^{\mathrm{m}_{1}} \mathrm{C}_{\mathrm{k}_{1}} \mathrm{p}_{1}^{\mathrm{k}_{1}}\left(1-\mathrm{p}_{1}\right)^{\mathrm{m}_{1}-\mathrm{k}_{1}}}{1-\left(1-\mathrm{p}_{1}\right)^{\mathrm{m}_{1}}}{ }^{\mathrm{k}_{1}} \mathrm{C}_{\mathrm{r}}(-1)^{3 \mathrm{r}} \frac{\left(1-\mathrm{e}^{-\mathrm{r} \beta_{1} \mathrm{t}}\right)}{\mathrm{r} \beta_{1}}\right]\right]
\end{aligned}
$$




$$
\begin{aligned}
& +\mathrm{R}_{2} \cdot \beta_{2} \cdot\left\{1-\exp \left\{\left[\alpha_{1} \sum_{\mathrm{k}_{1}=1}^{\mathrm{m}_{1}} \sum_{\mathrm{r}=1}^{\mathrm{k}_{1}} \sum_{\mathrm{J}=0}^{\mathrm{r}}(-1)^{3 \mathrm{r}-\mathrm{J}} \frac{{ }^{\mathrm{m}_{1}} \mathrm{C}_{\mathrm{k}_{1}} \mathrm{p}_{1}^{\mathrm{k}_{1}}\left(1-\mathrm{p}_{1}\right)^{\mathrm{m}_{1}-\mathrm{k}_{1}}}{1-\left(1-\mathrm{p}_{1}\right)^{\mathrm{m}_{1}}}\left({ }^{\mathrm{k}_{1}} \mathrm{C}_{\mathrm{r}}\right)\left({ }^{\mathrm{r}} \mathrm{C}_{\mathrm{J}}\right)\left(\frac{\beta_{1}}{\beta_{2}-\beta_{1}}\right)^{\mathrm{r}}\left(\frac{1-\mathrm{e}^{-\left[\mathrm{J} \beta_{2}+(\mathrm{r}-\mathrm{J}) \beta_{1}\right] \mathrm{t}}}{\mathrm{J} \beta_{2}+(\mathrm{r}-\mathrm{J}) \beta_{1}}\right)\right]\right.\right. \\
& \left.\left.+\alpha_{2}\left[\sum_{\mathrm{k}_{2}=1}^{\mathrm{m}_{2}} \sum_{\mathrm{s}=1}^{\mathrm{k}_{2}} \frac{{ }^{\mathrm{m}_{2}} \mathrm{C}_{\mathrm{k}_{2}} \mathrm{p}_{2}{ }^{\mathrm{k}_{2}}\left(1-\mathrm{p}_{2}\right)^{\mathrm{m}_{2}-\mathrm{k}_{2}}}{1-\left(1-\mathrm{p}_{2}\right)^{\mathrm{m}_{2}}}\left({ }^{\mathrm{k}_{2}} \mathrm{C}_{\mathrm{s}}\right)(-1)^{\mathrm{s}}\left(\frac{1-\mathrm{e}^{-\mu_{2} \mathrm{k}_{2} \mathrm{t}}}{\mu_{2} \mathrm{k}_{2}}\right)\right]\right\}\right\} \\
& -\mathrm{C}_{1} \cdot \frac{\frac{\alpha_{1}}{\beta_{1}}\left[\sum_{\mathrm{k}_{1}=1}^{\mathrm{m}_{1}} \frac{{ }^{\mathrm{m}_{1}} \mathrm{C}_{\mathrm{k}_{1}} \mathrm{p}_{1}{ }^{\mathrm{k}_{1}}\left(1-\mathrm{p}_{1}\right)^{\mathrm{m}_{1}-\mathrm{k}_{1}}}{1-\left(1-\mathrm{p}_{1}\right)^{\mathrm{m}_{1}}} \cdot \mathrm{k}_{1}\left(1-\mathrm{e}^{-\beta_{1} \mathrm{t}}\right)\right]}{-\beta_{1}\left[1-\exp \left[\alpha_{1} \sum_{\mathrm{k}_{1}=1}^{\mathrm{m}_{1}} \sum_{\mathrm{r}=1}^{\mathrm{k}_{1}} \frac{{ }^{\mathrm{m}_{1}} \mathrm{C}_{\mathrm{k}_{1}} \mathrm{p}_{1}{ }^{\mathrm{k}_{1}}\left(1-\mathrm{p}_{1}\right)^{\mathrm{m}_{1}-\mathrm{k}_{1}}}{1-\left(1-\mathrm{p}_{1}\right)^{\mathrm{m}_{1}}}{ }^{\mathrm{k}_{1}} \mathrm{C}_{\mathrm{r}}(-1)^{3 \mathrm{r}} \frac{\left(1-\mathrm{e}^{-\mathrm{r} \beta_{1} \mathrm{t}}\right)}{\mathrm{r} \beta_{1}}\right]\right]} \\
& -C_{2} \cdot \frac{\frac{\alpha_{1}}{\beta_{2}}\left(\sum_{k_{1}=1}^{\mathrm{m}_{1}} \frac{\mathrm{m}_{1}}{C_{\mathrm{k}_{1}} p_{1}^{\mathrm{k}_{1}}\left(1-\mathrm{p}_{1}\right)^{\mathrm{m}_{1}-\mathrm{k}_{1}}} \mathrm{k}_{1}\right)\left[\left(1-\mathrm{p}_{1}\right)^{\mathrm{m}_{1}}\right)\left[\left(1-\mathrm{e}^{-\beta_{2} \mathrm{t}}\right)+\frac{\beta_{2}}{\beta_{2}-\beta_{1}}\left(\mathrm{e}^{-\beta_{2} \mathrm{t}}-\mathrm{e}^{-\beta_{1} \mathrm{t}}\right)\right]+\frac{\alpha_{2}}{\beta_{2}}\left(\sum_{\mathrm{k}_{2}=1}^{\infty} \frac{{ }^{\mathrm{m}_{2}} \mathrm{C}_{\mathrm{k}_{2}} \mathrm{p}_{2}{ }^{\mathrm{k}_{2}}\left(1-\mathrm{p}_{2}\right)^{\mathrm{m}_{2}-\mathrm{k}_{2}}}{1-\left(1-\mathrm{p}_{2}\right)^{\mathrm{m}_{2}}} \mathrm{k}_{2}\right)\left(1-\mathrm{e}^{-\beta_{2} \mathrm{t}}\right)}{\beta_{2} \cdot\left\{1-\exp \left\{\left[\alpha_{1} \sum_{\mathrm{k}_{1}=1}^{\mathrm{m}_{1}} \sum_{\mathrm{r}=1}^{\mathrm{k}_{1}} \sum_{\mathrm{J}=0}^{\mathrm{r}}(-1)^{3 \mathrm{r}-\mathrm{J}} \frac{{ }^{\mathrm{m}_{1}} \mathrm{C}_{\mathrm{k}_{1}} \mathrm{p}_{1}^{\mathrm{k}_{1}}\left(1-\mathrm{p}_{1}\right)^{\mathrm{m}_{1}-\mathrm{k}_{1}}}{1-\left(1-\mathrm{p}_{1}\right)^{\mathrm{m}_{1}}}\left({ }^{\mathrm{k}_{1}} \mathrm{C}_{\mathrm{r}}\right)\left({ }^{\mathrm{r}} \mathrm{C}_{\mathrm{J}}\right)\left(\frac{\beta_{1}}{\beta_{2}-\beta_{1}}\right)^{\mathrm{r}}\left(\frac{1-\mathrm{e}^{-\left[\mathrm{J} \beta_{2}+(\mathrm{r}-\mathrm{J}) \beta_{1}\right] \mathrm{t}}}{\mathrm{J} \beta_{2}+(\mathrm{r}-\mathrm{J}) \beta_{1}}\right)\right]\right.\right.} \\
& \left.\left.+\alpha_{2}\left[\sum_{\mathrm{k}_{2}=1}^{\mathrm{m}_{2}} \sum_{\mathrm{s}=1}^{\mathrm{k}_{2}} \frac{{ }^{\mathrm{m}_{2}} \mathrm{C}_{\mathrm{k}_{2}} \mathrm{p}_{2}^{\mathrm{k}_{2}}\left(1-\mathrm{p}_{2}\right)^{\mathrm{m}_{2}-\mathrm{k}_{2}}}{1-\left(1-\mathrm{p}_{2}\right)^{\mathrm{m}_{2}}}\left({ }^{\mathrm{k}_{2}} \mathrm{C}_{\mathrm{s}}\right)(-1)^{\mathrm{S}}\left(\frac{1-\mathrm{e}^{-\beta_{2} \mathrm{k}_{2} \mathrm{t}}}{\beta_{2} \mathrm{k}_{2}}\right)\right]\right\}\right\}
\end{aligned}
$$

To verify the hessian matrix $\frac{\partial \mathrm{P}(\mathrm{t})}{\partial \beta_{1}}=0$ implies

$$
\begin{aligned}
& \frac{\partial \mathrm{P}(\mathrm{t})}{\partial \beta_{1}}=\mathrm{R}_{1} \cdot \beta_{1}\left[1-\exp \left[\alpha_{1} \sum_{\mathrm{k}_{1}=1}^{\mathrm{m}_{1}} \sum_{\mathrm{r}=1}^{\mathrm{k}_{1}} \frac{{ }^{\mathrm{m}_{1}} \mathrm{C}_{\mathrm{k}_{1}} \mathrm{p}_{1}{ }^{\mathrm{k}_{1}}\left(1-\mathrm{p}_{1}\right)^{\mathrm{m}_{1}-\mathrm{k}_{1}}}{1-\left(1-\mathrm{p}_{1}\right)^{\mathrm{m}_{1}}}{ }^{\mathrm{k}_{1}} \mathrm{C}_{\mathrm{r}}(-1)^{3 \mathrm{r}} \frac{\left(1-\mathrm{e}^{-\mathrm{r} \beta_{1} \mathrm{t}}\right)}{\mathrm{r} \beta_{1}}\right]\right] \\
& +\mathrm{R}_{2} \cdot \beta_{2} \cdot\left\{1-\exp \left\{\left[\alpha_{1} \sum_{\mathrm{k}_{1}=1}^{\mathrm{m}_{1}} \sum_{\mathrm{r}=1}^{\mathrm{k}_{1}} \sum_{\mathrm{J}=0}^{\mathrm{r}}(-1)^{3 \mathrm{r}-\mathrm{J}} \frac{{ }^{\mathrm{m}_{1}} \mathrm{C}_{\mathrm{k}_{1}} \mathrm{p}_{1}^{\mathrm{k}_{1}}\left(1-\mathrm{p}_{1}\right)^{\mathrm{m}_{1}-\mathrm{k}_{1}}}{1-\left(1-\mathrm{p}_{1}\right)^{\mathrm{m}_{1}}}\left({ }^{\mathrm{k}_{1}} \mathrm{C}_{\mathrm{r}}\right)\left({ }^{\mathrm{r}} \mathrm{C}_{\mathrm{J}}\right)\left(\frac{\beta_{1}}{\beta_{2}-\beta_{1}}\right)\left(\frac{1-\mathrm{e}^{-\left[\mathrm{J} \beta_{2}+(\mathrm{r}-\mathrm{J}) \beta_{1}\right] \mathrm{t}}}{\mathrm{J} \beta_{2}+(\mathrm{r}-\mathrm{J}) \beta_{1}}\right)\right]\right.\right. \\
& \left.\left.+\alpha_{2}\left[\sum_{\mathrm{k}_{2}=1}^{\mathrm{r}} \sum_{\mathrm{s}=1}^{\mathrm{m}_{2}} \frac{{ }_{\mathrm{m}_{2}} \mathrm{C}_{\mathrm{k}_{2}} \mathrm{p}_{2}{ }^{\mathrm{k}_{2}}\left(1-\mathrm{p}_{2}\right)^{\mathrm{m}_{2}-\mathrm{k}_{2}}}{1-\left(1-\mathrm{p}_{2}\right)^{\mathrm{m}_{2}}}\left({ }^{\mathrm{k}_{2}} \mathrm{C}_{\mathrm{s}}\right)(-1)^{\mathrm{s}}\left(\frac{1-\mathrm{e}^{-\mathrm{\mu}_{2} \mathrm{k}_{2} \mathrm{t}}}{\mu_{2} \mathrm{k}_{2}}\right)\right]\right\}\right\}
\end{aligned}
$$




$$
\begin{aligned}
& -\mathrm{C}_{1} \cdot \frac{\frac{\alpha_{1}}{\beta_{1}}\left[\sum_{\mathrm{k}_{1}=1}^{\mathrm{m}_{1}} \frac{{ }^{\mathrm{m}_{1}} \mathrm{C}_{\mathrm{k}_{1}} \mathrm{p}_{1} \mathrm{k}_{1}\left(1-\mathrm{p}_{1}\right)^{\mathrm{m}_{1}-\mathrm{k}_{1}}}{1-\left(1-\mathrm{p}_{1}\right)^{\mathrm{m}_{1}}} \cdot \mathrm{k}_{1}\left(1-\mathrm{e}^{-\beta_{1} \mathrm{t}}\right)\right]}{=\beta_{1}\left[1-\exp \left[\alpha_{1} \sum_{\mathrm{k}_{1}=1}^{\mathrm{m}_{1}} \sum_{\mathrm{r}=1}^{\mathrm{k}_{1}} \frac{{ }^{\mathrm{m}_{1}} \mathrm{C}_{\mathrm{k}_{1}} \mathrm{p}_{1}^{\mathrm{k}_{1}}\left(1-\mathrm{p}_{1}\right)^{\mathrm{m}_{1}-\mathrm{k}_{1}}}{1-\left(1-\mathrm{p}_{1}\right)^{\mathrm{m}_{1}}}{ }^{\mathrm{k}_{1}} \mathrm{C}_{\mathrm{r}}(-1)^{3 \mathrm{r}} \frac{\left(1-\mathrm{e}^{-\mathrm{r} \beta_{1} \mathrm{t}}\right)}{\mathrm{r} \beta_{1}}\right]\right]} \\
& -C_{2} \cdot \frac{\frac{\alpha_{1}}{\beta_{2}}\left(\sum_{\mathrm{k}_{1}=1}^{\mathrm{m}_{1}} \frac{{ }^{\mathrm{m}_{1}} \mathrm{C}_{\mathrm{k}_{1}} \mathrm{p}_{1}^{\mathrm{k}_{1}}\left(1-\mathrm{p}_{1}\right)^{\mathrm{m}_{1}-\mathrm{k}_{1}}}{1-\left(1-\mathrm{p}_{1}\right)^{\mathrm{m}_{1}}} \mathrm{k}_{1}\right)\left[\left(1-\mathrm{e}^{-\beta_{2} \mathrm{t}}\right)+\frac{\beta_{2}}{\beta_{2}-\beta_{1}}\left(\mathrm{e}^{-\beta_{2} \mathrm{t}}-\mathrm{e}^{-\beta_{1} \mathrm{t}}\right)\right]+\frac{\alpha_{2}}{\beta_{2}}\left(\sum_{\mathrm{k}_{2}=1}^{\infty} \frac{{ }^{\mathrm{m}_{2}} \mathrm{C}_{\mathrm{k}_{2}} \mathrm{p}_{2}^{\mathrm{k}_{2}}\left(1-\mathrm{p}_{2}\right)^{\mathrm{m}_{2}-\mathrm{k}_{2}}}{1-\left(1-\mathrm{p}_{2}\right)^{\mathrm{m}_{2}}} \mathrm{k}_{2}\right)\left(1-\mathrm{e}^{-\beta_{2} \mathrm{t}}\right)}{\beta_{2} \cdot\left\{1-\exp \left\{\left[\alpha_{1} \sum_{\mathrm{k}_{1}=1}^{\mathrm{m}_{\mathrm{r}}} \sum_{\mathrm{r}=1}^{\mathrm{k}_{1}} \sum_{\mathrm{J}=0}^{\mathrm{r}}(-1)^{3 \mathrm{r}-\mathrm{J}} \frac{{ }^{\mathrm{m}_{1}} \mathrm{C}_{\mathrm{k}_{1}} \mathrm{p}_{1}^{\mathrm{k}_{1}}\left(1-\mathrm{p}_{1}\right)^{\mathrm{m}_{1}-\mathrm{k}_{1}}}{1-\left(1-\mathrm{p}_{1}\right)^{\mathrm{m}_{1}}}\left({ }^{\mathrm{k}_{1}} \mathrm{C}_{\mathrm{r}}\right)\left({ }^{\mathrm{r}} \mathrm{C}_{\mathrm{J}}\right)\left(\frac{\beta_{1}}{\beta_{2}-\beta_{1}}\right)^{\mathrm{r}}\left(\frac{1-\mathrm{e}^{-\left[\mathrm{J} \beta_{2}+(\mathrm{r}-\mathrm{J}) \beta_{1}\right] \mathrm{t}}}{\mathrm{J} \beta_{2}+(\mathrm{r}-\mathrm{J}) \beta_{1}}\right)\right]\right.\right.}=0 \\
& \left.\left.+\alpha_{2}\left[\sum_{\mathrm{k}_{2}=1}^{\mathrm{m}_{2}} \sum_{\mathrm{s}=1}^{\mathrm{k}_{2}} \frac{{ }^{\mathrm{m}_{2}} \mathrm{C}_{\mathrm{k}_{2}} \mathrm{p}_{2}^{\mathrm{k}_{2}}\left(1-\mathrm{p}_{2}\right)^{\mathrm{m}_{2}-\mathrm{k}_{2}}}{1-\left(1-\mathrm{p}_{2}\right)^{\mathrm{m}_{2}}}\left({ }^{\mathrm{k}_{2}} \mathrm{C}_{\mathrm{s}}\right)(-1)^{\mathrm{s}}\left(\frac{1-\mathrm{e}^{-\beta_{2} \mathrm{k}_{2} \mathrm{t}}}{\beta_{2} \mathrm{k}_{2}}\right)\right]\right\}\right\}
\end{aligned}
$$

$$
\begin{aligned}
& \frac{\partial \mathrm{P}(\mathrm{t})}{\partial \beta_{2}}=0 \text { Implies: } \\
& \frac{\partial \mathrm{P}(\mathrm{t})}{\partial \beta_{2}}=\mathrm{R}_{1} \cdot \beta_{1}\left[1-\exp \left[\alpha_{1} \sum_{\mathrm{k}_{1}=1}^{\mathrm{m}_{1}} \sum_{\mathrm{r}=1}^{\mathrm{k}_{1}} \frac{{ }^{\mathrm{m}_{1}} \mathrm{C}_{\mathrm{k}_{1}} \mathrm{p}_{1}^{\mathrm{k}_{1}}\left(1-\mathrm{p}_{1}\right)^{\mathrm{m}_{1}-\mathrm{k}_{1}}}{1-\left(1-\mathrm{p}_{1}\right)^{\mathrm{m}_{1}}}{ }^{\mathrm{k}_{1}} \mathrm{C}_{\mathrm{r}}(-1)^{3 \mathrm{r}} \frac{\left(1-\mathrm{e}^{-\mathrm{r} \beta_{1} \mathrm{t}}\right)}{\mathrm{r} \beta_{1}}\right]\right] \\
& +\mathrm{R}_{2} \cdot \beta_{2} \cdot\left\{1-\exp \left\{\left[\alpha_{1} \sum_{\mathrm{k}_{1}=1}^{\mathrm{m}_{1}} \sum_{\mathrm{r}=1}^{\mathrm{k}_{1}} \sum_{\mathrm{J}=0}^{\mathrm{r}}(-1)^{3 \mathrm{r}-\mathrm{J}} \frac{{ }^{\mathrm{m}_{1}} \mathrm{C}_{\mathrm{k}_{1}} \mathrm{p}_{1}^{\mathrm{k}_{1}}\left(1-\mathrm{p}_{1}\right)^{\mathrm{m}_{1}-\mathrm{k}_{1}}}{1-\left(1-\mathrm{p}_{1}\right)^{\mathrm{m}_{1}}}\left({ }^{\mathrm{k}_{1}} \mathrm{C}_{\mathrm{r}}\right)\left({ }^{\mathrm{r}} \mathrm{C}_{\mathrm{J}}\right)\left(\frac{\beta_{1}}{\beta_{2}-\beta_{1}}\right)^{\mathrm{r}}\left(\frac{1-\mathrm{e}^{-\left[\mathrm{J} \beta_{2}+(\mathrm{r}-\mathrm{J}) \beta_{1}\right] \mathrm{t}}}{\mathrm{J} \beta_{2}+(\mathrm{r}-\mathrm{J}) \beta_{1}}\right)\right]\right.\right. \\
& \left.\left.+\alpha_{2}\left[\sum_{\mathrm{k}_{2}=1}^{\mathrm{m}_{2}} \sum_{\mathrm{s}=1}^{\mathrm{k}_{2}} \frac{{ }^{\mathrm{m}_{2}} \mathrm{C}_{\mathrm{k}_{2}} \mathrm{p}_{2}{ }^{\mathrm{k}_{2}}\left(1-\mathrm{p}_{2}\right)^{\mathrm{m}_{2}-\mathrm{k}_{2}}}{1-\left(1-\mathrm{p}_{2}\right)^{\mathrm{m}_{2}}}\left({ }^{\mathrm{k}_{2}} \mathrm{C}_{\mathrm{s}}\right)(-1)^{\mathrm{S}}\left(\frac{1-\mathrm{e}^{-\mu_{2} \mathrm{k}_{2} \mathrm{t}}}{\mu_{2} \mathrm{k}_{2}}\right)\right]\right\}\right\} \\
& -\mathrm{C}_{1} \cdot \frac{\frac{\alpha_{1}}{\beta_{1}}\left[\sum_{\mathrm{k}_{1}=1}^{\mathrm{m}_{1}} \frac{{ }^{\mathrm{m}_{1}} \mathrm{C}_{\mathrm{k}_{1}} \mathrm{p}_{1}{ }^{\mathrm{k}_{1}}\left(1-\mathrm{p}_{1}\right)^{\mathrm{m}_{1}-\mathrm{k}_{1}}}{1-\left(1-\mathrm{p}_{1}\right)^{\mathrm{m}_{1}}} \cdot \mathrm{k}_{1}\left(1-\mathrm{e}^{-\beta_{1} \mathrm{t}}\right)\right]}{\beta_{1}\left[1-\exp \left[\alpha_{1} \sum_{\mathrm{k}_{1}=1}^{\mathrm{m}_{1}} \sum_{\mathrm{r}=1}^{\mathrm{k}_{1}} \frac{{ }^{\mathrm{m}_{1}} \mathrm{C}_{\mathrm{k}_{1}} \mathrm{p}_{1}{ }^{\mathrm{k}_{1}}\left(1-\mathrm{p}_{1}\right)^{\mathrm{m}_{1}-\mathrm{k}_{1}}}{1-\left(1-\mathrm{p}_{1}\right)^{\mathrm{m}_{1}}}{ }^{\mathrm{k}_{1}} \mathrm{C}_{\mathrm{r}}(-1)^{3 \mathrm{r}} \frac{\left(1-\mathrm{e}^{-\mathrm{r} \beta_{1} \mathrm{t}}\right)}{\mathrm{r} \beta_{1}}\right]\right]}
\end{aligned}
$$




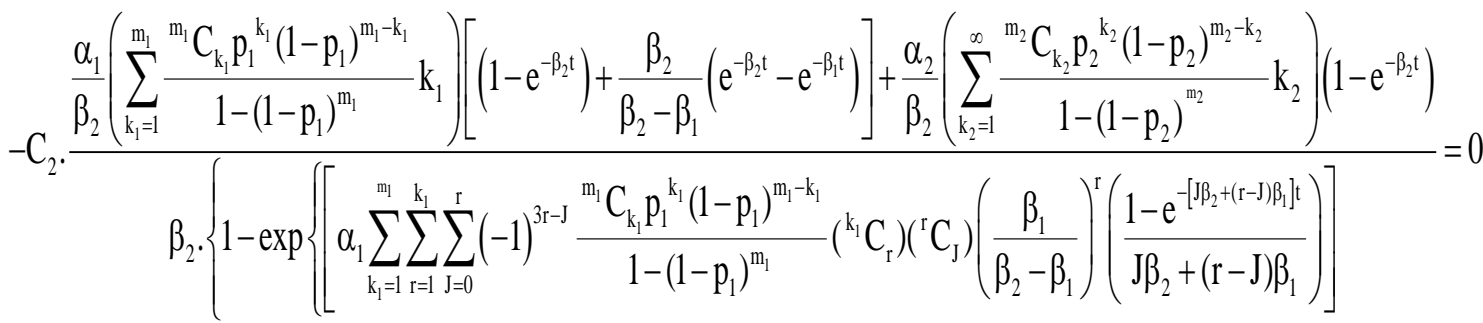

$$
\begin{aligned}
& \left.\left.\left.+\alpha_{2}\left[\sum_{k_{2}=1}^{\mathrm{m}_{2}} \sum_{\mathrm{s}=1}^{\mathrm{k}_{2}} \frac{{ }^{\mathrm{m}_{2}} \mathrm{C}_{\mathrm{k}_{2}} \mathrm{p}_{2}{ }^{\mathrm{k}_{2}}\left(1-\mathrm{p}_{2}\right)^{\mathrm{m}_{2}-\mathrm{k}_{2}}}{1-\left(1-\mathrm{p}_{2}\right)^{\mathrm{m}_{2}}}\left({ }^{\mathrm{k}_{2}} \mathrm{C}_{\mathrm{s}}\right)(-1)^{\mathrm{s}}\left(\frac{1-\mathrm{e}^{-\beta_{2} \mathrm{k}_{2} \mathrm{t}}}{\beta_{2} \mathrm{k}_{2}}\right)\right]\right\}\right\}\right\}
\end{aligned}
$$

The determinant of the Hessian matrix is,

$$
|\mathrm{D}|=\left|\begin{array}{ll}
\frac{\partial^{2} \mathrm{P}(\mathrm{t})}{\partial \beta_{1}{ }^{2}} & \frac{\partial^{2} \mathrm{P}(\mathrm{t})}{\partial \beta_{1} \partial \beta_{2}} \\
\frac{\partial^{2} \mathrm{P}(\mathrm{t})}{\partial \beta_{1} \partial \beta_{2}} & \frac{\partial^{2} \mathrm{P}(\mathrm{t})}{\partial \beta_{2}{ }^{2}}
\end{array}\right|<0
$$

Substituting the values of $\beta 1 *$ and $\beta 2 *$ in equation (6), we get the optimal value of the profit at given time $\mathrm{t}$ as

$$
\begin{aligned}
& \mathrm{P}^{*}(\mathrm{t})=\mathrm{R}_{1} \cdot \beta_{1}^{*}\left[1-\exp \left[\alpha_{1} \sum_{\mathrm{k}_{1}=1}^{\mathrm{m}_{1}} \sum_{\mathrm{r}=1}^{\mathrm{k}_{1}} \frac{{ }^{\mathrm{m}_{1}} \mathrm{C}_{\mathrm{k}_{1}} \mathrm{p}_{1}{ }^{\mathrm{k}_{1}}\left(1-\mathrm{p}_{1}\right)^{\mathrm{m}_{1}-\mathrm{k}_{1}}}{1-\left(1-\mathrm{p}_{1}\right)^{\mathrm{m}_{1}}}{ }^{\mathrm{k}_{1}} \mathrm{C}_{\mathrm{r}}(-1)^{3 \mathrm{r}} \frac{\left(1-\mathrm{e}^{-\mathrm{r} \beta_{1}{ }^{*} \mathrm{t}}\right)}{\mathrm{r} \beta_{1}^{*}}\right]\right] \\
& +\mathrm{R}_{2} \cdot \beta_{2}^{*} \cdot\left\{1-\exp \left\{\left[\alpha_{1} \sum_{\mathrm{k}_{1}=1}^{\mathrm{m}_{1}} \sum_{\mathrm{r}=1}^{\mathrm{k}_{1}} \sum_{\mathrm{J}=0}^{\mathrm{r}}(-1)^{3 \mathrm{r}-\mathrm{J}} \frac{{ }^{\mathrm{m}_{1}} \mathrm{C}_{\mathrm{k}_{1}} \mathrm{p}_{1}^{\mathrm{k}_{1}}\left(1-\mathrm{p}_{1}\right)^{\mathrm{m}_{1}-\mathrm{k}_{1}}}{1-\left(1-\mathrm{p}_{1}\right)^{\mathrm{m}_{1}}}\left({ }^{\mathrm{k}_{1}} \mathrm{C}_{\mathrm{r}}\right)\left({ }^{\mathrm{r}} \mathrm{C}_{\mathrm{J}}\right)\left(\frac{\beta_{1}{ }^{*}}{\beta_{2}{ }^{*}-\beta_{1} *}\right)^{\mathrm{r}}\left(\frac{1-\mathrm{e}^{-\left[\mathrm{J} \beta_{2}{ }^{*}(\mathrm{r}-\mathrm{J}) \beta_{1} * \mathrm{t}\right.}}{\mathrm{J} \beta_{2}{ }^{*}+(\mathrm{r}-\mathrm{J}) \beta_{1}{ }^{*}}\right)\right]\right.\right. \\
& \left.\left.+\alpha_{2}\left[\sum_{\mathrm{k}_{2}=1}^{\mathrm{m}_{2}} \sum_{\mathrm{s}=1}^{\mathrm{k}_{2}} \frac{{ }^{\mathrm{m}_{2}} \mathrm{C}_{\mathrm{k}_{2}} \mathrm{p}_{2}{ }^{\mathrm{k}_{2}}\left(1-\mathrm{p}_{2}\right)^{\mathrm{m}_{2}-\mathrm{k}_{2}}}{1-\left(1-\mathrm{p}_{2}\right)^{\mathrm{m}_{2}}}\left({ }^{\mathrm{k}_{2}} \mathrm{C}_{\mathrm{s}}\right)(-1)^{\mathrm{S}}\left(\frac{1-\mathrm{e}^{-\beta_{2}{ }^{*} \mathrm{k}_{2} \mathrm{t}}}{\beta_{2} * \mathrm{k}_{2}}\right)\right]\right\}\right\}
\end{aligned}
$$

\section{Arithmetical analysis}

In the current section, we exhibit the answer process during an arithmetical illustration.

Table 1. Arithmetical symbol of best value of $\beta_{1}{ }^{*}$ and $\beta_{2}{ }^{*}$

\begin{tabular}{|c|c|c|c|c|c|c|c|c|c|c|c|c|c|c|}
\hline $\mathrm{t}^{*}$ & $\mathrm{~m}_{1}$ & $\mathrm{~m}_{2}$ & $\mathrm{p}_{1}$ & $\mathrm{p}_{2}$ & $\alpha_{1} \#$ & $\alpha_{2} \#$ & $\mathrm{R}_{1}$ & $\mathrm{R}_{2}$ & $\mathrm{C}_{1}$ & $\mathrm{C}_{2}$ & $\beta_{1} * \$$ & $\beta_{2} * \$$ & $\mathrm{R}$ & $\mathrm{D}_{1}$ \\
\hline 0.6 & 6 & 5 & 0.3 & 0.4 & 0.4 & 0.3 & 0.6 & 0.7 & 0.2 & 0.3 & 3.498 & 3.380 & 3.015 & -0.564 \\
\hline 0.7 & 6 & 5 & 0.3 & 0.4 & 0.4 & 0.3 & 0.6 & 0.7 & 0.2 & 0.3 & 3.461 & 3.449 & 3.038 & -0.564 \\
\hline 0.8 & 6 & 5 & 0.3 & 0.4 & 0.4 & 0.3 & 0.6 & 0.7 & 0.2 & 0.3 & 3.426 & 3.473 & 3.045 & -0.564 \\
\hline 0.4 & 3 & 5 & 0.3 & 0.4 & 0.4 & 0.3 & 0.6 & 0.7 & 0.2 & 0.3 & 2.387 & 3.392 & 2.466 & -0.927 \\
\hline 0.4 & 4 & 5 & 0.3 & 0.4 & 0.4 & 0.3 & 0.6 & 0.7 & 0.2 & 0.3 & 2.973 & 3.475 & 2.829 & -0.927 \\
\hline 0.4 & 5 & 5 & 0.3 & 0.4 & 0.4 & 0.3 & 0.6 & 0.7 & 0.2 & 0.3 & 3.125 & 3.511 & 2.920 & -0.927 \\
\hline
\end{tabular}




\begin{tabular}{|c|c|c|c|c|c|c|c|c|c|c|c|c|c|c|}
\hline 0.4 & 6 & 2 & 0.3 & 0.4 & 0.4 & 0.3 & 0.6 & 0.7 & 0.2 & 0.3 & 3.293 & 3.180 & 2.863 & -0.656 \\
\hline 0.4 & 6 & 3 & 0.3 & 0.4 & 0.4 & 0.3 & 0.6 & 0.7 & 0.2 & 0.3 & 3.451 & 3.335 & 2.999 & -0.656 \\
\hline 0.4 & 6 & 4 & 0.3 & 0.4 & 0.4 & 0.3 & 0.6 & 0.7 & 0.2 & 0.3 & 3.876 & 3.568 & 3.271 & -0.656 \\
\hline 0.4 & 6 & 5 & 0.4 & 0.4 & 0.4 & 0.3 & 0.6 & 0.7 & 0.2 & 0.3 & 3.874 & 3.220 & 3.159 & -0.189 \\
\hline 0.4 & 6 & 5 & 0.5 & 0.4 & 0.4 & 0.3 & 0.6 & 0.7 & 0.2 & 0.3 & 3.942 & 3.224 & 3.190 & -0.189 \\
\hline 0.4 & 6 & 5 & 0.6 & 0.4 & 0.4 & 0.3 & 0.6 & 0.7 & 0.2 & 0.3 & 3.998 & 3.229 & 3.216 & -0.189 \\
\hline 0.4 & 6 & 5 & 0.3 & 0.5 & 0.4 & 0.3 & 0.6 & 0.7 & 0.2 & 0.3 & 3.013 & 3.442 & 2.827 & -0.496 \\
\hline 0.4 & 6 & 5 & 0.3 & 0.6 & 0.4 & 0.3 & 0.6 & 0.7 & 0.2 & 0.3 & 3.214 & 3.442 & 2.936 & -0.496 \\
\hline 0.4 & 6 & 5 & 0.3 & 0.7 & 0.4 & 0.3 & 0.6 & 0.7 & 0.2 & 0.3 & 3.458 & 3.442 & 3.060 & $\begin{array}{c}-0.496 \\
\end{array}$ \\
\hline 0.4 & 6 & 5 & 0.3 & 0.4 & 0.5 & 0.3 & 0.6 & 0.7 & 0.2 & 0.3 & 3.487 & 3.922 & 3.270 & -0.823 \\
\hline 0.4 & 6 & 5 & 0.3 & 0.4 & 0.6 & 0.3 & 0.6 & 0.7 & 0.2 & 0.3 & 3.564 & 3.965 & 3.326 & -0.823 \\
\hline 0.4 & 6 & 5 & 0.3 & 0.4 & 0.7 & 0.3 & 0.6 & 0.7 & 0.2 & 0.3 & 3.612 & 4.008 & 3.367 & -0.823 \\
\hline 0.4 & 6 & 5 & 0.3 & 0.4 & 0.4 & 0.3 & 0.6 & 0.7 & 0.2 & 0.3 & 3.721 & 3.938 & 3.391 & -0.270 \\
\hline 0.4 & 6 & 5 & 0.3 & 0.4 & 0.4 & 0.5 & 0.6 & 0.7 & 0.2 & 0.3 & 3.961 & 4.083 & 3.561 & $\begin{array}{c}-0.270 \\
\end{array}$ \\
\hline 0.4 & 6 & 5 & 0.3 & 0.4 & 0.4 & 0.6 & 0.6 & 0.7 & 0.2 & 0.3 & 4.010 & 4.153 & 3.611 & $\begin{array}{c}-0.270 \\
\end{array}$ \\
\hline 0.4 & 6 & 5 & 0.3 & 0.4 & 0.4 & 0.3 & 0.3 & 0.7 & 0.2 & 0.3 & 3.852 & 3.922 & 3.445 & -0.319 \\
\hline 0.4 & 6 & 5 & 0.3 & 0.4 & 0.4 & 0.3 & 0.4 & 0.7 & 0.2 & 0.3 & 3.875 & 3.944 & 3.465 & -0.319 \\
\hline 0.4 & 6 & 5 & 0.3 & 0.4 & 0.4 & 0.3 & 0.6 & 0.7 & 0.2 & 0.3 & 3.956 & 3.987 & 3.519 & -0.319 \\
\hline 0.4 & 6 & 5 & 0.3 & 0.4 & 0.4 & 0.3 & 0.6 & 0.3 & 0.2 & 0.3 & 3.851 & 3.907 & 3.438 & -0.645 \\
\hline 0.4 & 6 & 5 & 0.3 & 0.4 & 0.4 & 0.3 & 0.6 & 0.4 & 0.2 & 0.3 & 3.894 & 3.944 & 3.473 & -0.645 \\
\hline 0.4 & 6 & 5 & 0.3 & 0.4 & 0.4 & 0.3 & 0.6 & 0.6 & 0.2 & 0.3 & 3.976 & 4.050 & 3.554 & -0.645 \\
\hline 0.4 & 6 & 5 & 0.3 & 0.4 & 0.4 & 0.3 & 0.6 & 0.7 & 0.06 & 0.3 & 3.575 & 3.870 & 3.293 & -0.702 \\
\hline 0.4 & 6 & 5 & 0.3 & 0.4 & 0.4 & 0.3 & 0.6 & 0.7 & 0.07 & 0.3 & 3.591 & 3.893 & 3.310 & -0.702 \\
\hline 0.4 & 6 & 5 & 0.3 & 0.4 & 0.4 & 0.3 & 0.6 & 0.7 & 0.08 & 0.3 & 3.635 & 3.915 & 3.340 & -0.702 \\
\hline 0.4 & 6 & 5 & 0.3 & 0.4 & 0.4 & 0.3 & 0.6 & 0.7 & 0.2 & 0.07 & 3.845 & 3.907 & 3.436 & -0.139 \\
\hline 0.4 & 6 & 5 & 0.3 & 0.4 & 0.4 & 0.3 & 0.6 & 0.7 & 0.2 & 0.08 & 3.891 & 3.944 & 3.472 & -0.139 \\
\hline 0.4 & 6 & 5 & 0.3 & 0.4 & 0.4 & 0.3 & 0.6 & 0.7 & 0.2 & 0.09 & 3.934 & 3.980 & 3.506 & -0.139 \\
\hline
\end{tabular}

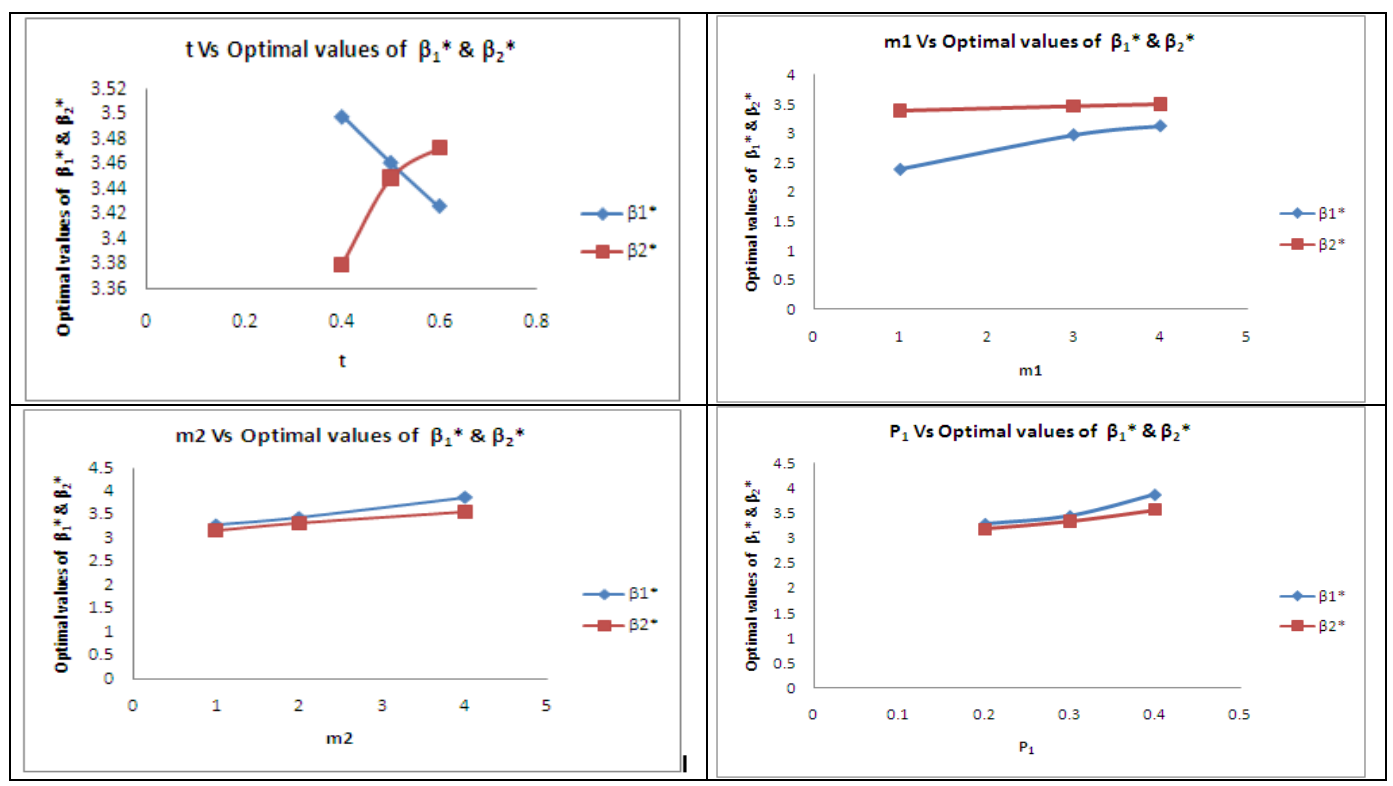




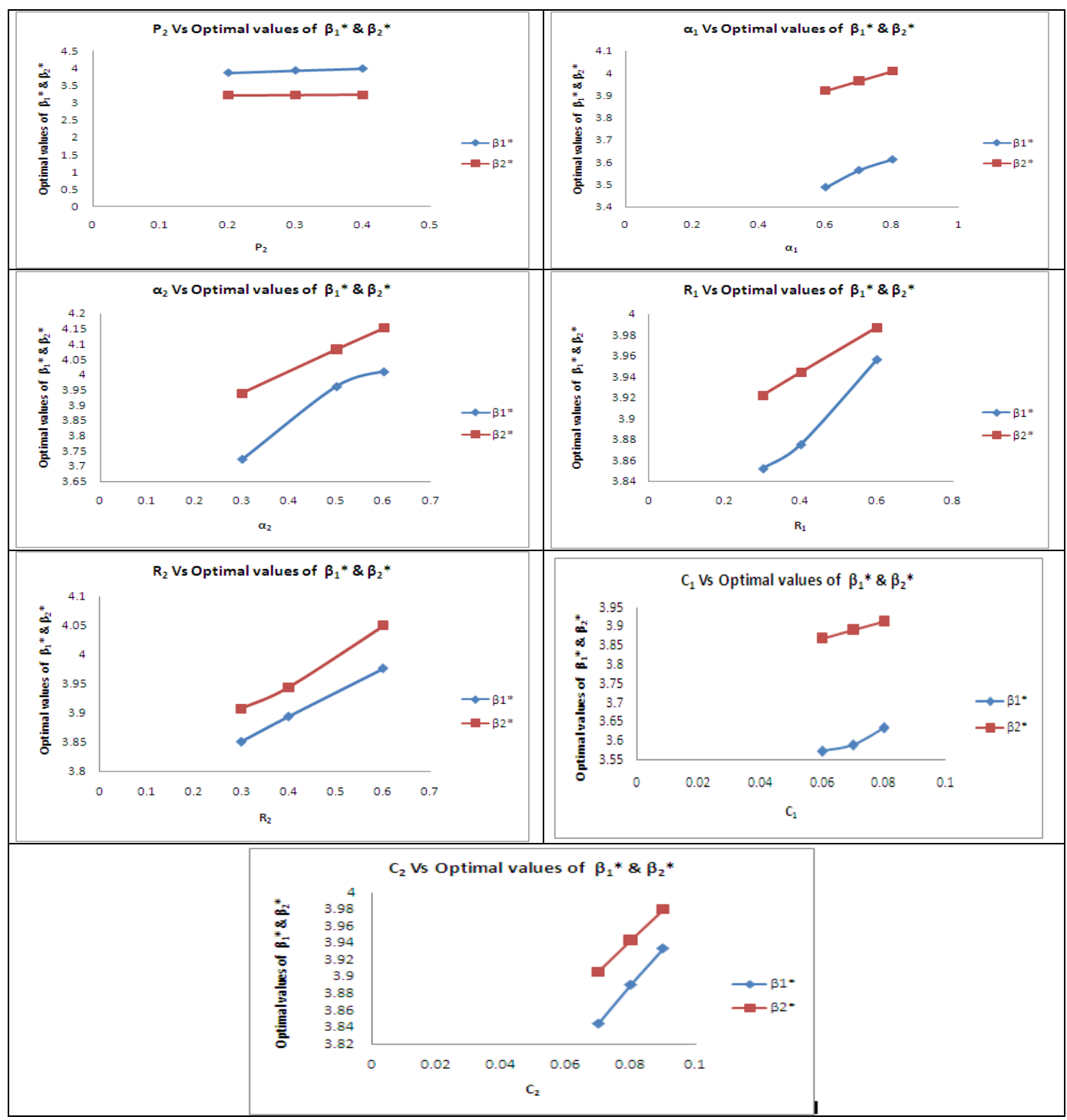

Figure 2. The optimal performance of a variety of considerations

\section{Sensitivity investigation}

The sensitivity examination of the Transmission Rate parameters $\beta 1^{*}$ and $\beta 2^{*}$, and the aggregate cost work $\mathrm{p}^{*}(\mathrm{t})$ are contemplated regarding the parameters $\mathrm{t}, \mathrm{m} 1, \mathrm{~m} 2, \mathrm{p} 1, \mathrm{p} 2, \alpha 1, \alpha 2$, $\mathrm{R} 1, \mathrm{R} 2, \mathrm{C} 1$ and $\mathrm{C} 2$. The following data has been considered for the sensitivity analysis.

$t=0.7 \mathrm{sec}$,

$m_{1}=1$,

$m_{2}=3, p_{1}=0.3, p_{2}=0.2, \alpha_{1}=0.5 \times 10^{4}$ packets $/ \mathrm{sec}, \alpha_{2}=0.4 \times 10^{4}$ packets $/ \mathrm{sec}, R_{1}=0.7$,

$R_{2}=0.6, C_{1}=0.4$ and $C_{2}=0.2$.

The execution measures are very influenced with the variety in time $(\mathrm{t})$ and the bunch estimate dissemination parameters of entries. As time (t) increments by $15 \%$ the normal number of bundles broadcast through the two cushions increments alongside the two transmitters and the landing rate of the parcels increments. As the group measure appropriation parameter $p$ 
increments to $15 \%$, the normal number of parcels broadcast through the two cradles increments alongside the two transmitters and the entry rate of the bundles increments. Overall investigation of the parameters mirrors that dynamic data transfer capacity distribution methodology for clog control immensely diminishes the mean postponement in correspondence and enhance influence excellence by lessening burstness in cushions.

Table 2. Sensitivity analysis

\begin{tabular}{|c|c|c|c|c|c|c|c|c|}
\hline \multirow[t]{2}{*}{ Parameter } & \multirow{2}{*}{$\begin{array}{c}\text { Performance } \\
\text { Measure }\end{array}$} & \multicolumn{7}{|c|}{$\%$ change in parameters } \\
\hline & & $-15 \%$ & $-10 \%$ & $-5 \%$ & 0 & $+5 \%$ & $+10 \%$ & $+15 \%$ \\
\hline \multirow{3}{*}{$\mathrm{t}=0.7$} & $\beta_{1}{ }^{*}$ & 4.013 & 4.052 & 4.087 & 4.105 & 4.126 & 4.152 & 4.178 \\
\hline & $\beta_{2} *$ & 3.950 & 3.970 & 3.991 & 4.011 & 4.031 & 4.051 & 4.071 \\
\hline & $\mathrm{R}$ & 3.581 & 3.605 & 3.628 & 3.643 & 3.660 & 3.683 & 3.697 \\
\hline \multirow{3}{*}{$\mathrm{P}_{1}=0.3$} & $\beta_{1}^{*}$ & 3.724 & 3.789 & 3.818 & 3.851 & 3.885 & 3.910 & 3.942 \\
\hline & $\beta_{2} *$ & 3.897 & 3.920 & 3.942 & 3.964 & 3.986 & 4.007 & 4.029 \\
\hline & $\mathrm{R}$ & 3.442 & 3.479 & 3.501 & 3.524 & 3.547 & 3.566 & 3.589 \\
\hline \multirow{3}{*}{$\mathrm{P}_{2}=0.2$} & $\beta_{1}^{*}$ & 3.896 & 3.924 & 3.952 & 3.976 & 3.998 & 4.018 & 4.036 \\
\hline & $\beta_{2}^{*}$ & 3.888 & 3.923 & 3.957 & 3.991 & 4.024 & 4.057 & 4.090 \\
\hline & $\mathrm{R}$ & 3.508 & 3.535 & 3.561 & 3.585 & 3.608 & 3.630 & 3.651 \\
\hline \multirow{3}{*}{$\alpha_{1}=0.5$} & $\beta_{1} *$ & 3.862 & 3.889 & 3.922 & 3.942 & 3.958 & 3.982 & 4.003 \\
\hline & $\beta_{2}{ }^{*}$ & 3.856 & 3.880 & 3.905 & 3.929 & 3.953 & 3.977 & 4.001 \\
\hline & $\mathrm{R}$ & 3.481 & 3.502 & 3.526 & 3.545 & 3.561 & 3.581 & 3.600 \\
\hline \multirow{3}{*}{$\alpha_{2}=0.4$} & $\beta_{1}{ }^{*}$ & 3.796 & 3.823 & 3.849 & 3.878 & 3.892 & 3.918 & 3.947 \\
\hline & $\beta_{2}{ }^{*}$ & 3.763 & 3.794 & 3.825 & 3.855 & 3.885 & 3.915 & 3.945 \\
\hline & $\mathrm{R}$ & 3.413 & 3.438 & 3.462 & 3.487 & 3.505 & 3.529 & 3.554 \\
\hline \multirow{3}{*}{$\mathrm{R}_{1}=0.7$} & $\beta_{1} *$ & 3.781 & 3.804 & 3.831 & 3.862 & 3.886 & 3.911 & 3.937 \\
\hline & $\beta_{2} *$ & 3.803 & 3.827 & 3.851 & 3.875 & 3.899 & 3.922 & 3.946 \\
\hline & $\mathrm{R}$ & 3.425 & 3.445 & 3.466 & 3.489 & 3.509 & 3.529 & 3.550 \\
\hline \multirow{3}{*}{$\mathrm{R}_{2}=0.6$} & $\beta_{1}{ }^{*}$ & 3.751 & 3.787 & 3.812 & 3.841 & 3.874 & 3.898 & 3.921 \\
\hline & $\beta_{2} *$ & 3.876 & 3.910 & 3.943 & 3.976 & 4.009 & 4.042 & 4.074 \\
\hline & $\mathrm{R}$ & 3.445 & 3.474 & 3.499 & 3.525 & 3.552 & 3.576 & 3.600 \\
\hline \multirow{3}{*}{$\mathrm{C}_{1}=0.4$} & $\beta_{1}{ }^{*}$ & 3.851 & 3.898 & 3.925 & 3.968 & 3.987 & 4.019 & 4.041 \\
\hline & $\beta_{2} *$ & 3.854 & 3.879 & 3.903 & 3.927 & 3.951 & 3.974 & 3.998 \\
\hline & $\mathrm{R}$ & 3.476 & 3.505 & 3.526 & 3.554 & 3.572 & 3.592 & 3.613 \\
\hline \multirow{3}{*}{$\mathrm{C}_{2}=0.2$} & $\beta_{1} *$ & 3.471 & 3.492 & 3.524 & 3.552 & 3.581 & 3.603 & 3.634 \\
\hline & $\beta_{2}{ }^{*}$ & 3.967 & 3.396 & 3.426 & 3.455 & 3.483 & 3.512 & 3.540 \\
\hline & $\mathrm{R}$ & 3.127 & 3.160 & 3.193 & 3.214 & 3.240 & 3.263 & 3.285 \\
\hline \multirow{4}{*}{$\mathrm{m}_{1}=1$} & & $-75 \%$ & $-50 \%$ & $-25 \%$ & 0 & $+25 \%$ & $+50 \%$ & $+75 \%$ \\
\hline & $\beta_{1} *$ & 3.487 & 3.503 & 3.521 & 3.548 & 3.561 & 3.578 & 3.591 \\
\hline & $\beta_{2}{ }^{*}$ & 3.415 & 3.427 & 3.440 & 3.452 & 3.464 & 3.477 & 3.489 \\
\hline & $\mathrm{R}$ & 3.146 & 3.159 & 3.174 & 3.193 & 3.204 & 3.219 & 3.230 \\
\hline \multirow{4}{*}{$\mathrm{m}_{2}=3$} & $\beta_{1}^{*}$ & 3.202 & 3.239 & 3.261 & 3.286 & 3.307 & 3.331 & 3.364 \\
\hline & $\beta_{2}{ }^{*}$ & 3.253 & 3.299 & 3.344 & 3.389 & 3.433 & 3.476 & 3.518 \\
\hline & $\mathrm{R}$ & 2.927 & 2.966 & 2.997 & 3.030 & 3.060 & 3.091 & 3.127 \\
\hline & $\mathrm{R}$ & 3.425 & 3.445 & 3.466 & 3.489 & 3.509 & 3.529 & 3.550 \\
\hline
\end{tabular}

\section{Conclusion}

The current article deal with a good and fresh correspondence arranges to demonstrate with mass landings having two-phase coordinate entries. Here it is expected that the messages arrive straightforwardly to the main cushion and second cradle which are associated pair. Advance it is expected that the communication is changed over into an irregular number of parcels and put away in cushions for forward transmission. The landing forms in both the cradles are portrayed with compound Poisson binomial procedures. With appropriate cost contemplations, the ideal working approaches of the correspondence organize are additionally determined. Through 
mathematical representations the affectability of the adjustments in input parameters and expenses on the ideal working arrangements is likewise examined. This correspondence organize is much helpful for execution assessment, control and observing of correspondence systems at information/voice transmissions, satellite interchanges, LLAN, WAN planning and web suppliers. This correspondence organize model can likewise be stretched out to nonMarkovian change forms which require assist examinations.

\section{References}

[1] M. V. Ramasundari, K. Srinivasa Rao, P. Srinivasa Rao, and P.S.Suresh varma, "Three node communication network model with dynamic bandwidth allocation and non homogeneous poisson arrivals," International journal of Computer Applications, vol.31, No.1, pp.19-27, (2011) DOI: 10.5120/3789-5217

[2] K.Srinivasa Rao, M.V.Ramasundari, P.Srinivasa Rao, and P.S.Sureshvarma, "Three node communication network model with modified phase type transmission under DBA having NHP arrivals," International journal of Computer Engineering, vol.4, no.1, pp.17-29, (2011)

[3] K. Srinivasa Rao, M.Govinda Rao, and P.Chandra Sekhar "Studies on interdependent tandem queuing models with modified phase type service and state dependent service rates," International Journal of Computer applications, vol.3, issue 4, pp.319-330, (2013)

[4] P.SureshVarma, K.Srinivasa Rao, and P.V.G.D.Prasad Reddy-, "A communication network with random phases of transmission," Indian Journal of Mathematics and Mathematical Sciences, vol.3, no.1, pp.39- 46, (2007)

[5] N.Thirupathi Rao, K.Srinivasa Rao, and P.Srinivasa Rao, "Optimal traffic flow in tandem communication networks," International Journal of Control and Automation, vol.11, no.12, pp.35-46, (2018)

[6] N.Thirupathi Rao, K.Srinivasa Rao, Kuda Nageswara Rao, and P.Srinivasa Rao, "Transient analysis of a two node tandem communication network with two stage compound poisson binomial bulk arrivals and DBA," International Journal of Computer Applications, vol.96, no.25, pp:19-31, (2014) 